where $C_{1}=1-\gamma, \gamma=\lim \left(1+\frac{1}{2}+\frac{1}{3}+\cdots+\frac{1}{n}-\log n\right):$ Euler's constant

$$
C_{r}=\frac{1}{r}\left(\frac{1}{2^{r}}+\frac{1}{3^{r}}+\frac{1}{4^{r}}+\cdots\right) \quad(r=2,3, \cdots) \text {. }
$$

The values of $S_{r}=\frac{1}{1^{r}}+\frac{1}{2^{r}}+\frac{1}{3^{r}}+\cdots$ and $\gamma$ were taken from Stieltjes' table. ${ }^{1}$ Part of the calculation was done with the assistance of Mr. E. V. HANKAM on an IBM (602-A type) calculating punch. Uhler's radix table was used for getting the antilog of $\log \Gamma$. The values $\Gamma\left(\frac{1}{3}\right)$ and $\Gamma\left(\frac{2}{3}\right)$ were required for calculating the power series coefficients of Bessel functions of order $\frac{1}{3}$ and of functions related to them.

The values were checked by the identity

$$
\begin{array}{rrrrrrr}
\sqrt{3} \Gamma\left(\frac{1}{3}\right) \Gamma\left(\frac{2}{3}\right)=2 \pi & & & & & \\
\Gamma\left(\frac{1}{3}\right)=2.67893 & 85347 & 07747 & 63365 & 56929 & 410 \\
\Gamma\left(\frac{2}{3}\right)=1.35411 & 79394 & 26400 & 41694 & 52880 & 282 \\
\log \Gamma\left(\frac{1}{3}\right)=.98542 & 06469 & 27767 & 06918 & 71740 & 370 \\
\log \Gamma\left(\frac{2}{3}\right)=.30315 & 02751 & 47523 & 56867 & 58628 & 174
\end{array}
$$

B. ZONDEK

Watson Scientific Computing Laboratory

New York, N. Y.

${ }^{1}$ H. T. DAvis, Tables of Higher Mathematical Functions. v. II, The.Principia Press, 1935, p. 244.

\title{
Modification of a Method for Calculating Inverse Trigonometric Functions
}

The 605 programming that I gave recently ${ }^{1}$ fails for arguments near $2^{-1}$. The reason for this failure is that the double angle formulations used multiply round-off errors until they are intolerably large. These formulations were originally introduced to assure that $\cos 2 \theta$ depend on both $\sin \theta$ and $\cos \theta$. Upon closer examination it was found that it is only necessary that $\cos 2 \theta$ depend on $\sin \theta$, hence we may use

$$
\cos 2 \theta=1-2 \sin ^{2} \theta .
$$

The use of the above formula and

$$
\sin 2 \theta=2 \sin \theta \cos \theta
$$

avoids the errors mentioned and is just as easily programmed for the 605 .

U. S. Naval Ordnance Plant

R. L. LA FARA

Indianapolis 18 , Indiana

${ }^{1}$ Richard L. LA FARA, $M T A C$, v. 8, 1954, p. 132-139. 